\title{
An unexpected side effect: Wartenberg syndrome related to the use of splint during carpal tunnel syndrome treatment
}

\author{
Azize Serçe, Ebru Karaca Umay, Özgür Zeliha Karaahmet, Fatma Aytül Çakcı \\ Department of Physical Medicine and Rehabilitation, Dışkapı Ylldırım Beyazıt Training and Research Hospital, Ankara, Turkey
}

Received: December 12, 2016 Accepted: March 02, 2017 Published online: June 19, 2017

\begin{abstract}
Isolated superficial radial neuropathy is a sensorial mononeuropathy and a rare clinical condition. In case of trapping of the sensorial branch of the radial nerve, symptoms such as burning type pain at proximal forearm and hand dorsoradial, hypoesthesia, numbness and tingling at hand dorsum and thumb radial side are observed. Muscular weakness and trophic changes are not seen, and electrophysiological assessment revealed normal motor nerve conduction values. Therefore, hand motor functions are not affected. The etiology of the isolated superficial radial nerve neuropathy is often associated with repeated use of wrist watches, forearm fractures, use of handcuffs, repetitive activities. In this report, we present a 59-year-old female case of isolated superficial radial nerve neuropathy diagnosed by electrophysiological evaluation who was treated for two months with carpal tunnel syndrome with altered symptoms and increased numbness. This is the first case of superficial radial nerve neuropathy following the splint use in the literature.
\end{abstract}

Keywords: Carpal tunnel syndrome; neuropathy; splint; Wartenberg.

Isolated superficial radial neuropathy (also called the Wartenberg Syndrome or Cheiralgia Paresthetica) is a sensory mononeuropathy and a rare clinical condition. ${ }^{[1,2]}$ It was first described in 1922 by Stopford ${ }^{[3]}$ and, since it has a superficial course, trapping may occur anywhere along the forearm.

The etiology of the isolated superficial radial nerve neuropathy is often associated with repeated use of wrist watches, falling, forearm fractures, surgical operations such as fracture fixation and arthroscopic procedures, steroid injections, acupuncture, use of handcuffs, repetitive supination, pronation, ulnar flexion activities, compression of extensor carpi radialis and/or brachioradialis tendons, presence of neuroma, lipoma or ganglion cyst in the wrist as well as exposure to excess cold. ${ }^{[1,4,5]}$ In addition, the presence of diabetes mellitus was reported to provide basis for development of the disease. ${ }^{[1,6,7]}$ However, no cases secondary to use of splints during carpal tunnel syndrome treatment have been reported in the literature. A therapeutic, non-invasive method that we frequently use in our daily practice is what has led to this situation.

In this report, we present an initial case of superficial radial neuropathy occurring following splint use due to carpal tunnel syndrome.

\section{CASE REPORT}

A 59-year-old female patient presented to our electrophysiology laboratory with the complaint of numbness in the right hand and a preliminary diagnosis of advanced carpal tunnel syndrome.

The numbness in her hand was present for approximately one year. The numbness that was previously present during the night, started occurring also during the day and spread to the first three fingers and, thus, the patient presented to our clinic two months ago. The patient who was referred to the electrophysiology laboratory was given resting splint holding the hand and the wrist in the neutral position with the diagnosis of mild carpal tunnel syndrome.

Corresponding author: Azize Serçe, MD. Dışkapı Yıldırım Beyazıt Ĕğitim ve Araştırma Hastanesi, Fiziksel Tıp ve Rehabilitasyon Kliniği, 06110 Dışkapı, Ankara, 
Table 1. Bilateral sensory nerve conduction study

\begin{tabular}{lcccc}
\hline Nerve/Sites & $\begin{array}{c}\text { Peak latency } \\
(\mathrm{ms})\end{array}$ & $\begin{array}{c}\text { Amplitude } \\
(\mu \mathrm{V})\end{array}$ & $\begin{array}{c}\text { Distance } \\
(\mathrm{cm})\end{array}$ & $\begin{array}{c}\text { Velocity } \\
(\mathrm{m} / \mathrm{s})\end{array}$ \\
\hline Right median-Digit II & 2.70 & 61.8 & 11 & 40.7 \\
Left median-Digit II & 2.55 & 65.1 & 11 & 43.1 \\
Right ulnar-Digit V & 2.45 & 65.0 & 11 & 44.9 \\
Left ulnar-Digit V & 2.55 & 63.4 & 11 & 43.8 \\
Right radial-Thumb & 2.40 & 7.1 & 9 & 37.5 \\
Left radial-Thumb & 2.25 & 26.2 & 9.5 & 42.2 \\
\hline
\end{tabular}

The patient used the splint day and night for two months and her numbness showed partial relief. However, the nature of numbness changed and she particularly had numbness in the thumb. Along with numbness, she recently described pain starting from the right hand, going up as far as the shoulder. While the numbness was intermittent initially, it gained continuity for the last one year. No morning stiffness was reported by the patient.

She had no known history of metabolic or endocrine disease, previous trauma, operation and/or cervical discopathy. Except the non-steroidal anti-inflammatory drugs (NSAIDs), she was using for the last 15 days; however, she had no regular drug use.

Her physical examination revealed an overall swelling in the right hand dorsal and sensitivity on dorsal hand upon palpation. Individual assessment of the finger joints showed no sensitivity or swelling suggesting arthritis. The articular range of motion was full in the cervical, shoulder, elbow, and wrist joints. Sensory examination revealed no loss of senses in the upper and lower extremities other than in the dorsal side of the thumb. No reduction was observed in the muscular strengths. Deep tendon reflexes were normal in the whole body.

Electrophysiology examination revealed a slower rate of nerve conduction and a lower amplitude for the radial nerve as achieved antidromically from the first digit. Needle electroneuromyography (ENMG) showed no results of axon damage in the extensor incidis, extensor digitorum communis, innerved by the radial nerve, or in the abductor pollicis brevis muscles innerved by the median nerve (Table 1 ).

Since there were no findings to suggest carpal tunnel syndrome in the patient's electrophysiological assessment, the splint use was discontinued; however NSAID use was continued and twice daily cold applications were added to treatment.

Diagnostic laboratory examinations revealed normal values for the inflammatory parameters, erythrocyte sedimentation rate, C-reactive protein, whole blood profile, anti-cyclic citrulline protein, anti-nuclear antibody and thyroid functions tests, B12 vitamin levels, and biochemical parameters such as renal function tests.

Two-way direct radiography showed no peculiarity other than soft tissue swelling. To exclude an early-stage inflammatory joint disease, hand magnetic resonance imaging (MRI) was performed which showed soft tissue edema and minimal fluid increase in the dorsal wrist. No bone marrow edema or bone erosion was observed.

At the time of investigations, (10-13 days after discontinuing use of splint), the patient reported that her pain was decreased. Swelling and sensitivity of the hand also decreased, but the feeling of numbness continued. The patient received a total of 15 sessions of cold application and transcutaneous electrical nerve stimulation for the treatment of superficial radial neuropathy. Follow-up visit six months later showed no complaints of numbness, and the nerve conduction study revealed that the radial nerve sensorial conduction completely returned to normal.

\section{DISCUSSION}

The radial nerve, originating from the C5-T1 root of the spinal cord and the posterior cord of the brachial plexus, innervates the brachioradialis, extensor carpi radialis longus, and brevis muscles and, then, divides into superficial and deep branches at the level of the forearm. While the deep branch innervates the supinator, forearm and hand extensor muscles, the superficial branch goes down to the hand dorsal ${ }^{[6]}$ and branches into the dorsal digital nerves in the proximal interphalangeal joint of the middle finger, thumb and index finger. ${ }^{[3,4]}$ The superficial branch of the radial nerve, due to its anatomic location, is vulnerable to compression from trauma, masses, and constriction from the fascia connecting the brachioradialis and extensor carpi radialis longus. This nerve runs under cover of the brachioradialis in the forearm. 
Eight centimeters proximal to the tip of the radial styloid, the nerve pierces the fascia medial to the brachioradialis to lie dorsal to the extensor tendons. Specifically, in pronation, the brachioradialis and the extensor carpi radialis longus compress the nerve.

In case of trapping of the sensorial branch of the radial nerve at the wrist level, symptoms such as intermittent or persistent burning type pain at proximal forearm and hand dorsoradial; hypoesthesia, numbness, and tingling at hand dorsum and thumb radial face are observed, depending on the duration of trapping. ${ }^{[1,8]}$ Muscular weakness and trophic changes are not observed; electrophysiological assessment revealed normal motor nerve conduction values. Thus, hand motor functions are not affected. ${ }^{[9]}$ In our case, after starting using the splint day and night, symptoms such as numbness, tingling and burning-type pain spreading toward the arm on the hand dorsal, being more marked in the thumb, which was intermittent initially, but subsequently became persistent, were observed. The second ENMG showed normal motor nerve conduction other than the sensorial involvement in the radial nerve.

Diseases such as De Quervain tenosynovitis, carpal tunnel syndrome, cervical discopathy, and brachial plexopathy that should be considered in the differential diagnosis that could lead to these symptoms were excluded through physical examination and electrophysiological assessment, while hand swelling and sensitivity were excluded by radiological and laboratory evaluations.

In our case, these differential diagnoses were excluded, and it was thought that the cause of these symptoms was radial nerve neuropathy due to splint use. In addition, we consider that the regression of symptoms upon discontinuation of splint use supports our theory. In the literature, elimination of the etiological factor as well as electrotherapy were reported to be effective. ${ }^{[1,8,10]}$ As in case of a peripheral nerve trap neuropathy, epineural blood flow and axonal transport is reduced due to early-stage ischemic block, leading to transient conduction problems, and elimination of the compression and conservative treatment make this condition reversible. ${ }^{[4]}$ Also, in our case, elimination of the etiological factor and two sessions of electrotherapy achieved completely normal nerve conduction after six months.

There are many studies reporting that use of stabilization procedures such as splints, elastic bandage, and bracing may lead to neuropathy in the peripheral nerves and may even cause severe functional disabilities and, particularly, there are many studies involving peroneal trap neuropathy among the lower extremity neuropathies and adequate attention has been given to this topic. As a result of inaccurate use of stabilization and immobilization tools, neuropathy may occur, particularly in the superficial nerves. ${ }^{[1]}$ The use of splints is recommended in the treatment of carpal tunnel syndrome, the most common type of trap neuropathy, as an established, practical treatment method without side effects in contrary to medication. ${ }^{[12]}$

In conclusion, when the use of splints is preferred as the treatment for carpal tunnel syndrome, one should keep in mind that this may lead to another trap neuropathy, as in our first case in the literature, and such patients should be followed with respect to potential isolated superficial neuropathy.

\section{Declaration of conflicting interests}

The authors declared no conflicts of interest with respect to the authorship and/or publication of this article.

\section{Funding}

The authors received no financial support for the research and/or authorship of this article.

\section{REFERENCES}

1. Politylo J, Decina PA, Lopes AA. Superficial radial neuropathy secondary to intravenous infusion at the wrist: a case report. The Journal of the CCA1993;37:92-6.

2. Wartenberg R. Neuritis produced by a wristlet watch. Lancet 1992;1:993-4.

3. Shetty P, Sirasanagandla SR, Dsouza MR. Possible Entrapment of Sensory Branch of Radial Nerve by Split Brachioradialis Tendon: An Anatomical Variation. IJHSR. 2014;4:204-7.

4. Hu SY, Choi JG, Son BC. Cheiralgiaparesthetica:an isolated neuropathy of the superficial branch of the radial nerve. Available from: http://www.thenerve.net/. [Accessed: September, 2015]

5. Xu S, Wang L, Cooper E, Zhang M, Manheimer E, Berman $B$, et al. Adverse events of acupuncture: a systematic review of case reports. Evid Based Complement Alternat Med 2013;2013:581203.

6. Demiryürek BE, Emre U, Demirel EA, Taşçılar FN, Atasoy HT, Ortancil Ö, et al. Radial sinir nöropatilerinin retrospektif değerlendirilmesi. İstanbul Med J2015;16:116-8.

7. Dang AC, Rodner CM. Unusual compression neuropathies of the forearm, part I: radial nerve. J Hand Surg Am 2009;34:1906-14.

8. Beredjiklian PK, Doumas C, Bozentka DJ, Steinberg DR. Compression neuropathies of the upper extremity. Philadelphia: Turner White Communications; 2011.

9. Akkaya N, Özcan HR, Gökalan Kara I, Sahin F. Bilateral isolated cut of sensory branch of radial nerve. Ulus Travma Acil Cerrahi Derg 2013;19:186-8. 
10. Lanzetta M, Foucher G. Entrapment of the superficial branch of the radial nerve (Wartenberg's syndrome). A report of 52 cases. Int Orthop 1993;17:342-5.

11. Craig A. Entrapment neuropathies of the lower extremity.
PM R 2013;5:31-40.

12. Page MJ, Massy-Westropp N, O'Connor D, Pitt V. Splinting for carpal tunnel syndrome. Cochrane Database Syst Rev 2012;7:CD010003. 